\title{
A Partition Formula for the Integer Coefficients of the Theta Function Nome
}

\author{
By Helaman Rolfe Pratt Ferguson, Dale E. Nielsen and Grant Cook
}

\begin{abstract}
In elliptic function theory, the nome $q$ can be given as a power series in $\epsilon$ with integer coefficients, $q=\Sigma_{n} \geqslant 0 \delta_{n} \epsilon^{4 n+1}$. Heretofore, the first 14 coefficients were calculated with considerable difficulty. In this paper, an explicit and general formula involving partitions is given for all the $\delta_{n}$. A table of the first 59 of these integers is given. The table is of number-theoretical interest as well as useful for calculating complete and incomplete elliptic integrals.
\end{abstract}

It has been pointed out almost everywhere [1]-[8] that the complete and incomplete elliptic integrals of the first and second kind can be calculated using the rapidly convergent theta functions. For example, the complete elliptic integral of the first kind, with modulus $k$, has the expressions, [5],

$$
\begin{aligned}
K(k) & =\int_{0}^{\pi / 2} \frac{d \theta}{\sqrt{1-k^{2} \sin ^{2} \theta}}=\frac{\pi}{2}\left(1+2 \sum_{n \geqslant 1} q^{n^{2}}\right)^{2} \\
& =\frac{\pi}{2}\left(1+4 \sum_{n \geqslant 1} \frac{q^{n}}{1+q^{2 n}}\right) .
\end{aligned}
$$

The nome $q$ is a function of the modulus $k$ via the intermediary $\epsilon$,

$$
\epsilon=\frac{1}{2}\left[\frac{1-\left(k^{\prime}\right)^{1 / 2}}{1+\left(k^{\prime}\right)^{1 / 2}}\right], \quad k^{\prime}=\left(1-k^{2}\right)^{1 / 2},
$$

where (Weierstrass [6])

$$
q=q(\epsilon)=\sum_{n \geqslant 0} \delta_{n} \epsilon^{4 n+1}
$$

The first few integer coefficients, $\delta_{n}, \delta_{0}=1, \delta_{1}=2, \delta_{2}=15, \delta_{3}=150, \delta_{4}=$ $1707, \ldots$, have been calculated, the first four by Weierstrass [6], six by MilneThomson [7], fourteen by Lowan, et al. [8]. In this paper, we give a completely general formula for all of these integers $\delta_{n}$ and calculate the first 59. These match exactly with those of [6], [7], [8].

THEOREM. Let the nome $q, \epsilon$ and $\delta_{n}, n \geqslant 1$, be as defined above. Then the integers

Received May 28, 1974; revised November 25, 1974.

AMS(MOS) subject classifications (1970). Primary 30A10, 33A25; Secondary 05A17, 10A40, 33-04.

Key words and phrases. Elliptic integrals, theta functions, nome, partitions, reversion.

Copyright $\mathbb{0} 1975$, American Mathematical Society 
TABLE 1

$$
\epsilon(q)=\sum_{k \geq 0} c_{k} q^{4 k+1}
$$

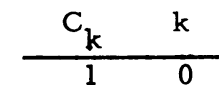

$\begin{array}{rl}-2 & 1\end{array}$

52

$-10$

18

$-32$

55

$-90$

144

$-226$

346

$-522$

777

$-1138$

1648

$-2362$

3348

$-4704$

6554

$-9056$

12425

$-16932$

22922

$-30848$

41282

$-54946$

72768

$-95914$

125842

$-164402$

213901

$-277204$

357904

$-460448$

590330

$-754368$

960948

$-1220370$

1545306

$-1951258$

2457152

$-3086112$

3866271

$-4831786$

6024144

$-7493554$

9300676

$-11518784$

14236130

$-17558850$

21614512 \begin{tabular}{rr}
$\mathrm{C}_{k}$ & $\mathrm{k}$ \\
\hline-26556084 & 51
\end{tabular}

$32566736 \quad 52$

$-39865632 \quad 53$

$48714496 \quad 54$

$-59425460 \quad 55$

$72370451 \quad 56$

$-87991906 \quad 57$

$106815482 \quad 58$

$-129465152 \quad 59$

$156680498 \quad 60$

$-189337106 \quad 61$

$228470640 \quad 62$

$-275304480 \quad 63$

$331282178 \quad 64$

$-398105538 \quad 65$

$477778404 \quad 66$

$-572657866 \quad 67$

$685514048 \quad 68$

$-819598930 \quad 69$

$978726474 \quad 70$

$-1167365856 \quad 71$

$1390748755 \quad 72$

$-1654993826 \quad 73$

$1967251104 \quad 74$

$-2335868000 \quad 75$

$2770581364 \quad 76$

$-3282739616 \quad 77$

$3885557834 \quad 78$

$-4594412154 \quad 79$

$5427179280 \quad 80$

$-6404625958 \quad 81$

$7550857344 \quad 82$

$-8893832810 \quad 83$

$10465956914 \quad 84$

$-12304758144 \quad 85$

$14453668032 \quad 86$

$-16962912538 \quad 87$

$19890533666 \quad 88$

$\begin{array}{r}-23303559744 \quad 89 \\ \hline\end{array}$

$27279342357 \quad 90$

$-31907085620 \quad 91$

$37289594562 \quad 92$

$-43545269554993$

$50810383492 \quad 94$

$-59241680384 \quad 95$

$69019335360 \quad 96$

$-80350328386 \quad 97$

$93472287408 \quad 98$

$-108657859786 \quad 99$

$126219686290 \quad 100$

(5)

$$
\delta_{n}=\sum_{1 \leqslant k \leqslant n}(-1)^{k} \sum^{*} \frac{(4 n+k) !}{(4 n+1) ! a_{1} ! \ldots a_{n} !} C_{1}^{a_{1}} \ldots C_{n}^{a_{n}},
$$

where $\Sigma^{*}$ is a summation over all the integer partitions $k=\Sigma_{1 \leqslant s \leqslant n} a_{s}, n=$ $\Sigma_{1 \leqslant s \leqslant n} s a_{s}, a_{s} \geqslant 0$. The $C_{k}$ are integers satisfying the recursion relation 
TABLE 2

$$
q(\epsilon)=\sum_{n \geq 0} \delta_{n} \epsilon^{4 n+1}
$$$$
\text { (2) }
$$

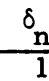

n

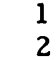

2
15

150

1707

20910

268616

3567400

48555069

673458874

9481557398

135119529972

1944997539623

28235172753886

412850231439153

6074299605748746

89857589279037102

1335623521633805028

19936473955587624656

298710089390201812048

4490774010052865283744

67720348285795481957568

1024048736429572060655319

155245378.51637363478060414

235895735639949246721659678

3592055029374068300374296756

54804356948531406780686077335

837668718981768081882232416270

12825003353647588436048753554935

196661581715188108128970795004550

3020038525632669791764002713422532

46440290652254605592799214895101504

715038427200862813001136930265147512

11022543326741896130999268335183824336

170106631417212663570292309396189783082

2627963818429706645211842908688374293300

40639684325725031991118847449107074979172

629057442741114268349966425119727665581336

9745798971459876443490275061762301063120623

151116397169795848764748621445190045537049166

2345060743702465972443964863972690339335580295

36418974504781217231871247380322076634437243526

566000002327718606520661245108793975375803955227

8802486794330300502560080205926854680407227244446

136987427943746069763667154538164819216291647222299

2133189839056434593163452664751723335632221518780078

33238368972758722951581782375851915672575296334699144

518203119165380946481537680006811067385208271890196520

8083508347684372357034150759458280957643776575928292964

126162240900543525003409858218743000597507890709208990152

1970058936472178165658457810603802393218322368577430279357

30778014840073879593583180632414116424005881087226151493994

481066708903723582902283009313154592694417730034725440142805

7522557895177030497054105691715628210384957408473671745338098

117683064927025633752081377689230738120581991161302571300341950

1841805068163667424373785303397419166787012535392792974110785668

28836865945637549391397672719750764886865336155791435146300051614 451669686692109094028753360935093143169400258806.504175758417989996 7077117970879174771151304161914657388532532100161812093166648499271

\begin{tabular}{l}
7 \\
\hline 0 \\
1 \\
2 \\
3 \\
4 \\
5 \\
6 \\
7 \\
8 \\
9 \\
10 \\
11 \\
12 \\
13 \\
14 \\
15 \\
16 \\
17 \\
18 \\
19 \\
20 \\
21 \\
22 \\
23 \\
24 \\
25 \\
26 \\
27 \\
28 \\
29 \\
30 \\
31 \\
32 \\
33 \\
34 \\
35 \\
36 \\
37 \\
38 \\
39 \\
40 \\
41 \\
42 \\
43 \\
44 \\
45 \\
46 \\
47 \\
48 \\
49 \\
50 \\
51 \\
52 \\
53 \\
54 \\
55 \\
\end{tabular}




$$
C_{k}=\delta_{k}^{m(m+1)}-2 \sum_{0<s \leqslant \sqrt{k}} C_{k-s^{2}},
$$

where $C_{0}=1, C_{1}=-2, C_{2}=5, C_{3}=-10, C_{4}=18, \ldots$, and $\delta_{k}^{m(m+1)}$ is one if $k$ is a product of consecutive integers, $k=m(m+1)$ and zero otherwise.

Proof. Weierstrass [6, p. 275] showed that

$$
\left(1+2 \sum_{k \geqslant 1} q^{4 k^{2}}\right) \epsilon=\sum_{k \geqslant 1} q^{(2 k-1)^{2}} .
$$

After performing the power series division to solve for $\epsilon$, we find

$$
\epsilon=\sum_{k \geqslant 0} C_{k} q^{4 k+1},
$$

where the integers $C_{k}$ satisfy the recursion relation (5). All we have to do now is solve for $q$ in Eq. (8). We do this by the technique of series reversion. For example, Jolley [9] and Van Orstand [10] give the first seven and twelve coefficients, respectively, for power series reversion. Finding the general term in this development is an old combinatorial problem first solved by McMahon [11]. Note that Eq. (8) as a power series has zero coefficients except for every fourth term. This fact simplifies McMahon's formula to Eq. (5).

Previous calculations of the $\delta_{n}$ [6], [7], [8] were done by rewriting (6) to give

$$
q=\epsilon+\sum_{k \geqslant 1} 2 \epsilon q^{(2 k)^{2}}-q^{(2 k+1)^{2}} .
$$

This expression can be used as a recursion relation to eliminate $q$ on the right-hand side. It is clear from this that the $\delta_{n}$ are integers.

Table I gives the integers $C_{k}, k=0,1,2, \ldots, 100$. The recursion relation defining them only requires $\sqrt{k}$ terms for each $k$, hence calculation of the $C_{k}$ is not time consuming. However, constructing [13] Table II, $\delta_{n}, n=0,1,2, \ldots, 58$, involves generating all the partitions of $n$ for each $n$. The number $p(n)$ of partitions of $n$ is $O\left(n^{-1} e^{\alpha \sqrt{n}}\right)$, Rademacher [12]. The decimal representation of $\delta_{58}$ has 67 digits, involved 715, 220 partitions, and took over four hours on an IBM 7030 [13]. The operations were performed by variable-length multiple-precision STRAP subroutines.

We thank the referee for pointing out that Rauch [14, Section 7], using his version of the above theorem, checked the coefficients of Lowan, et al. [8]. Computationally, the two techniques are identical, e.g., both involve sums over partitions.

\footnotetext{
Department of Mathematics

Brigham Young University

Provo, Utah 84602

Institute for Plasma Research

Stanford University

Stanford, California 94305

Department of Physics and Astronomy

Science Computation Center

Brigham Young University

Provo, Utah 84602
} 
1. E. T. WhitTAKER \& G. N. WATSON, $A$ Course of Modern Analysis, 4th ed., Cambridge Univ. Press, New York, 1927, Sect. 21.8, pp. 485-486.

2. E. JAHNKE \& F. EMDE, Tables of Functions, with Formulae and Curves, 4th ed., Dover, New York, 1945 (note that on page 74 the fifth coefficient is misprinted, it should be 1707 instead of 1701 - this has been corrected in some later editions). MR 7, 485 .

3. L. M. MILNE-THOMSON, Jacobian Elliptic Functions Tables. A Guide to Practical Computation with Elliptic Functions and Integrals Together with Tables of $\operatorname{sn} u, \operatorname{cn} u, \operatorname{dn} u, Z(u)$, Dover, New York, 1950. MR 13, 987.

4. E. HILLE, Analytic Function Theory. Vol. II, Introductions to Higher Math., Ginn, Boston, Mass., 1962, p. 160. MR 34 \#1490.

5. I. S. GRADŠTEİN \& I. M. RYŽIK, Tables of Integrals, Series and Products, 4th ed., Fizmatgiz, Moscow, 1963; English transl., Academic Press, New York, 1965, pp. 921-925. MR 28 \#5198; 33 \#5952.

6. A. WEIERSTRASS, “Zur Theorie der Elliptischen Funktionen,” Werke, v. 2, 1895, pp. 275-276.

7. L. M. MILNE-THOMSON, "Ten decimal table of the nome $q$," J. London Math. Soc., v. 5, 1930, pp. 148-149.

8. A. N. LOWAN, G. BLANCH \& W. HORENSTEIN, "On the inversion of the $q$-series associated with Jacobian elliptic functions,” Bull. Amer. Math. Soc., v. 48, 1942, pp. 737-738. MR 4, 90.

9. L. B. W. JOLLEY, Summation of Series, 2nd rev. ed., Dover Books on Advanced Math., Dover, New York, 1961, pp. 30-31. MR 24 \#B511.

10. C. E. VAN ORSTRAND, "Reversion of power series," Philos. Mag., v. 19, 1910, pp. 366-376.

11. M. McMAHON, "On the general term in the reversion of series," Bull. Amer. Math. Soc., v. 3, 1894, pp. 170-172.

12. H. RADEMACHER, "On the partition function," Proc. London Math. Soc., v. 43, 1937, pp. 241-254.

13. We thank the BYU Scientific Computation Center and their resurrected IBM 7030 (an old STRETCH) for doing the extensive number crunching of which Table II is a distillation.

14. LOUIS M. RAUCH, "Some general inversion formulae for analytic functions," Duke Math. J., v. 18, 1951, pp. 131-146. MR 12, 813. 